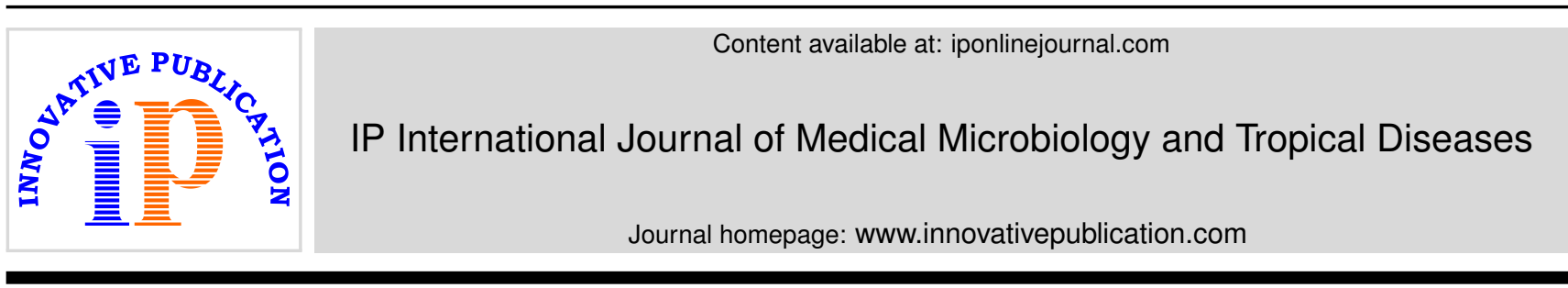

Original Reserach Article

\title{
Occurrence of brucellosis in patients of pyrexia of unknown origin
}

\author{
Pradeep Kumar $^{1}$, Shweta R Sharma ${ }^{1} *$, Umar Farooq ${ }^{1}$, Sudhir Singh ${ }^{1}$, \\ Vasundhara Sharma ${ }^{1}$, Imran Ahamad ${ }^{1}$, Shivendra Mohan ${ }^{1}$ \\ ${ }^{1}$ Dept. of Microbiology, Teerthanker Mahaveer Medical College \& Research Centre, Moradabad, Uttar Pradesh, India
}

\section{A R T I C L E I N F O}

\section{Article history:}

Received 24-03-2020

Accepted 01-06-2020

Available online 06-07-2020

\section{Keywords:}

PUO

Brucellosis

Agglutination

Prevalence

\begin{abstract}
A B S T R A C T
Background: Brucellosis is the most common bacterial zoonotic disease worldwide.It is mainly occupational disease reported in farmers, slaughter house workers, animal handlers and meat inspectors. This disease is endemic in many parts of Asia, including India. Brucellosis is an important, but neglected major cause of pyrexia of unknown origin(PUO). Diagnosis of brucellosis is difficult. Hence the medical and laboratory staff needs to be alert to recognize this condition.

Objective: To assess the possible risk factors associated with human brucellosis.To study frequency of human brucellosis in relation to occupation, age and sex.

Materials and Methods: From September (2018) to November (2019) 102 Serum samples of PUO cases were analysed for brucellosis. All serum samples were analysed by slide agglutination method using standard specific brucella antigen.

Result: We observed that human brucellosis is present in PUO patients. The total number of brucellosis positive cases were found to be $14(13.72 \%)$ out of 102 PUO cases. Among positive cases, males were 8 $(57.14 \%)$ and females were $6(42.85 \%)$. Highest brucellosis positive cases were seen in age-group between $15-30$ years $6(42.85 \%)$ followed by $31-45$ years $5(35.71 \%)$, $46-60$ years $2(14.28 \%) \&>601(7.14 \%)$ in PUO patients.

Conclusion: With the growing number of brucellosis cases detected in India. Brucellosis is the fast emerging as a public health threat and also due to limited diagnostics leading to underreporting. The study also highlights the innovative and cost effective methods for rapid and early reporting and ruling out brucellosis in PUO cases.

(C) 2020 Published by Innovative Publication. This is an open access article under the CC BY-NC license (https://creativecommons.org/licenses/by-nc/4.0/)
\end{abstract}

\section{Introduction}

Brucellosis is an old, infectious and common zoonosis whose causative agents are Gram-negative bacteria from the Brucella genus. Brucellosis is transmitted by goats, pigs, camels, sheep, buffalo and cows. Brucellosis is more common in children than in adults. Non-specific symptoms of brucellosis include fever, chills, restlessness, headache, fatigue and weakness. ${ }^{1}$ One of the most common symptoms of this disease is bone involvement that is seen in over half of the affected people. The association between Brucella and brucellosis was first discovered by an army surgeon

\footnotetext{
* Corresponding author.

E-mail address: drshwetamicro@gmail.com (S. R. Sharma).
}

named David Bruce. ${ }^{2}$ He isolated bacteria from the spleen of patients in Malta from 1886 to 1887 . Brucellosis is still one of the most common zoonosis in the world and more than 500000 cases each year are diagnosed with the disease, most of whom live in developing countries. ${ }^{3}$ The number of brucellosis cases are over 1 million people in some countries where brucellosis is endemic. Centers for Disease Control and Prevention has classified brucellosis as a category B bioterrorism agent due to its easy transmission.

The disease cycle can be acute, sub-acute or chronic. ${ }^{4}$ Four different Brucella species, i.e. Brucella suis (B. suis), Brucella melitensis (B. melitensis), Brucella abortus (B. abortus) and Brucella canis can be pathogenic to humans. ${ }^{2}$ Among these species, B. melitensis is the 
most contagious species, followed by B. abortus and B. suis. Brucella species are intracellular, obligate and Gramnegative coccobacilli and are not able to produce spores. ${ }^{5}$ Brucella species have no classic virulence genes encoding capsules, plasmids, pili or exotoxins, and because of factors that cause the bacteria to acquire resistance to phagocytes and increase the proliferation of phagocytic cells, they are relatively less known than other pathogenic bacteria. ${ }^{4,5}$

Each year more than 500000 people acquire brucellosis and most of them live in Asian countries including Syria, Iraq, Iran, India and Mongolia. The overall prevalence has been reported to be high but the prevalence in China and South Korea is low. ${ }^{6}$ However, the disease remains endemic in some areas of the two countries. Brucellosis is more common in men due to their greater activity than women and children. ${ }^{4}$

\subsection{Ethics and consent to participate statement}

Ethics approval was obtained from TMMC Moradabad institutional Ethics committee (TMMC-IEC) Ref. No. TMMC \& RC/IEC/18-19/080. Written consent/assent was sought from all individuals before enrolment.

\section{Material and Methods}

The study was conducted in serology section of Microbiology, Department of Teerthanker Mahaveer Medical College \& Research Centre, Moradabad. 102 clinical samples received as per inclusion and exclusion criteria were processed over the period of 11 months from September (2018) to November (2019). Patients with Widal test, MP test and dengue test positive were kept in exclusion criteria for PUO patients. Blood was collected \& tested for antibrucella antibodies against B. abortus antigen by slide agglutination method.

\section{Result}

The total number of brucellosis positive cases were found to be $14(13.72 \%)$ out of 102 PUO cases. Among positive cases, males were $8(57.14 \%)$ and females were $6(42.85 \%)$. Highest brucellosis positive cases were seen in age-group between $15-30$ years $6(42.85 \%)$ followed by $31-45$ years $5(35.71 \%)$, $46-60$ years $2(14.28 \%) \&>601(7.14 \%)$ in PUO patients.

Table 1: Distributation of positive and negative cases

\begin{tabular}{ll} 
Test & Number of PUO cases \\
Brucella -A Positive & 14 \\
Brucella-A Negative & 88 \\
Total & 102 \\
\hline
\end{tabular}

Showing a total 102 bloodsamples were processed from suspected case of pyrexia of unknown origin (PUO) patients in which 14 were positive and 88 were negative for brucellosis.

Table 2: Sex-wise distribution

\begin{tabular}{llll}
\hline Gender & Brucella & Percentage \% & Total PUO cases \\
Male & 8 & 7.84 & 44 \\
Female & 6 & 5.88 & 58 \\
Total & 14 & 13.72 & 102 \\
\hline
\end{tabular}

A total 102 blood samples were processed from suspected case of pyrexia of unknown origin (PUO) patients in which 14 were positive for brucella species among them 8 were males and 6 were females.

Table 3: Age-wise distribution

\begin{tabular}{lll}
\hline Age & Male & Female \\
$15-30$ & 3 & 3 \\
$31-45$ & 3 & 2 \\
$46-60$ & 1 & 1 \\
$>60$ & 1 & 0 \\
Total & 8 & 6 \\
\hline
\end{tabular}

The maximum numbers of patients were in the age group $15-30$ years $(6.0)$ followed by $31-45$ years $(5.0), 46-60$ years (2.0) \& >60 years (1.0) in PUO patients.

Highest prevalence was seen in age group between 15-30 years $6(42.85 \%)$ followed by $31-45$ years $5(35.71 \%)$, 4660 years $2(14.28 \%) \&>60$ years $1(7.14 \%)$ in PUO patients.

\section{aNIMAL HANDLERS HOUSE HOLD}

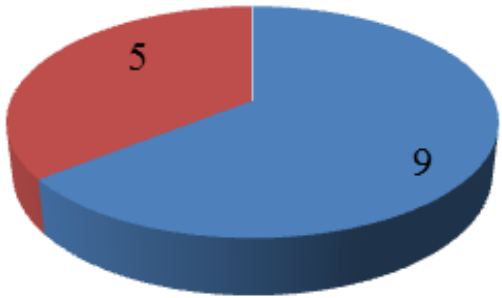

Fig. 1: Occupation - wise positive cases

Out of 14 brucella positive cases, 9 were animal handlers and 5 were household.

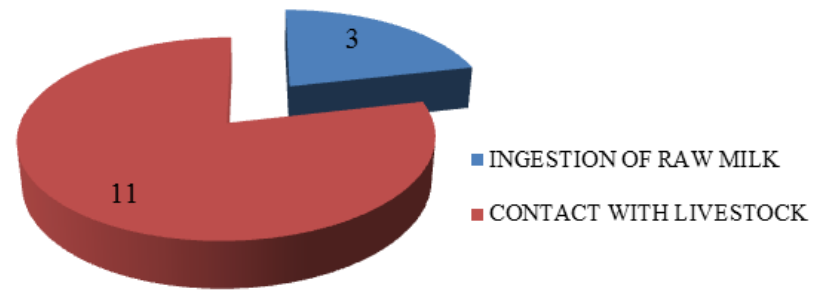

Fig. 2: Correlation of risk factors in brucella 
Table 4: Distribution of brucellosis according to gender and age

\begin{tabular}{llllll}
\hline Age & Puo cases & Male & Female & Total positive & Percentage\% \\
$15-30$ & 57 & 19 & 38 & 6 & 42.85 \\
$31-45$ & 28 & 17 & 11 & 5 & 35.71 \\
$46-60$ & 11 & 5 & 6 & 2 & 14.28 \\
$>60$ & 6 & 3 & 3 & 1 & 7.14 \\
Total & 102 & 44 & 58 & 14 & $100 \%$ \\
\hline
\end{tabular}

Risk factors such contact with livestock was found to be more clearly linked with brucellosis. 11 out of 14 cases were in contact with livestock.

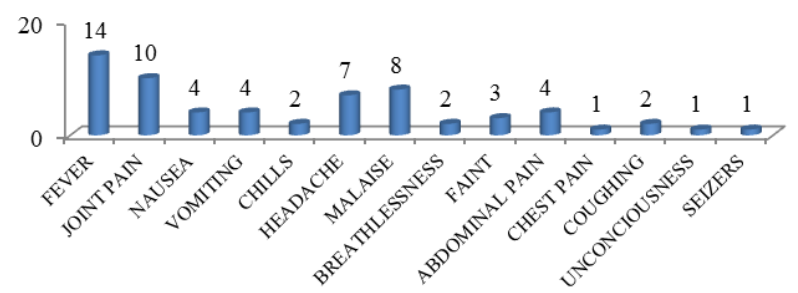

Fig. 3: Clinical symptoms in brucellosis positive PUO patients

Showing majority of brucellosis positive patients presented with symptoms of fever, joint pain followed by malaise, headache, nausea, vomiting, abdominal- pain, faint, chills, breathlessness, chest pain, unconsciousness \& seizers.

\section{Discussion}

In this study, we investigated the incidence of brucellosis in pyrexia of unknown origin from patients attending TMMC \& RC Moradabad (UP). In our study total 102 blood samples of PUO patients were screened for brucellosis test 14 patients were showed to be brucellosis positive and 88 were negative.

In this study prevalence of human brucellosis in pyrexia of unknown origin patients is $13.72 \%$. These are similar to recent study Rahamathulla MP et al. analyzed the seroprevalance in different regions of Saudi Arabia, the maximum prevalence of $20 \%$ and $18.3 \%$ were seen in northern as well as southern area respectively by $14 \%$ in the central and eastern region each, $11.6 \%$ in the western region. ${ }^{7}$ In contrast Patil DP et al (2016) analyzed the sero positivity of brucellosis in PUO suspected cases in north Karnataka region was to be $189(5.1 \%)$. Males are more effected due to their professional activities. This study indicated that the male constituted the majority $8(57.14 \%)$ of the patients by females $6(42.85 \%){ }^{3}$ In contrast' Prakash $\mathrm{P}$ et al (2012) which show that prevalence is more among females $58(53.70 \%)$ as compare to males $50(46.29 \%)$ in 108 brucellosis positive cases. Highest brucellosis infection was showed in age group between 15-30 years $42.85 \%$, followed by $35.71 \%$ in the range of $31-45$ years age group,
$14.28 \%$ in $46-60$ and $7.14 \%$ were $>60$ years of age. ${ }^{8}$ The present study correlates with Mangalgi SS et al (2012) which showed that majority of the patients were seen in age group 21-30 years $28(24.56 \%)$ followed by $0-10$ years 19 (16.66\%), 11-20 years $21(18.42 \%), 31-40$

years $(19.29 \%), 41-50$ years $11(9.6 \%), 51-60$ years 8 $(7.01 \%),>60$ years $5(4.38 \%) .{ }^{1}$ In contrast Prakash P et al (2012) which show that maximum positive were seen in age group 31-40 years. $8.77 \%$ were between $11-20$ year, come after $24.07 \%$ in the scale of $21-30$ years, $26.85 \%$ in $31-40$ years, $16.66 \%$ in $41-50$ years, $10.18 \%$ in $51-60$ years and $13.88 \%$ were $>60$ years of age. ${ }^{8}$ Out of 14 brucella positive cases, $9(64.28 \%)$ were animal handlers. $5(35.71 \%)$ patients were household. These similar were studies done by Muloki $\mathrm{HN}$ et al (2018) who also reported maximum positivity rate in animal handlers $145(57.76 \%) .{ }^{9}$ But in contrast Metri BC et al (2011) which show that maximum positive were seen in household $187(49.21 \%)$ followed by farmers $115(30.26 \%)$, shepherd $17(4.47 \%)$, others $61(16.05 \%) .^{10}$

These finding were similar to the studies done by Muloki HN et al who also shown brucellosis seropositivity to be highly associated with consumption of unpasteurized milk and milk products. ${ }^{9}$ This study clearly shows livestock rearing to be firmly linked with human brucellosis. The highest symptoms with fever (100\%) followed by joint pain $(71.42 \%)$. Patients had complaints of malaise $(57.14 \%)$, headache $(50 \%)$, vomiting $(28.57 \%)$, nausea $(28.57 \%)$, abdominal pain $(28.57 \%)$. Rest of patients had complaints of chills (14.28\%), breathlessness (14.28\%), coughing (14.28\%), chest pain $(7.14 \%)$, unconsciousness (7.14\%), seizure $(7.14 \%)$.

\section{Conclusion}

Finding in this study show clearly that the sero-prevalence of human brucellosis among patients with prolonged fever is high $(13.72 \%)$. Rearing livestock and consumption of raw milk and milk products were factors associated with brucellosis. Strategies to control animal brucellosis and raising awareness about the consumption of raw milk and milk products are necessary to reduce the incidence of human infection. Innovative methods to sensitize the community on brucellosis and cost effective methods to control animal brucellosis are required \& recommended. 


\section{Source of Funding}

None.

\section{Conflict of Interest}

None.

\section{References}

1. Mangaligi SS, Sajjan AG, Mohite ST. Brucellosis: A cause for pytexia of unknown origin. Int J Biomed Res. 2012;3(3):2054-58.

2. Bano Y, Lone SA. Brucellosis: An Economically Important Infection. J Med Microbiodiagn. 2015;04:1-8.

3. Shetty PC, Patil DP, Ajantha GS, Shubhada C, Jain PA, Kalabhavi A, et al. Trend of human brucellosis over a decade at tertiary care centre in North Karnataka. India J Med Microb. 2016;34(4):427-32.

4. Manish K, Puran C, Rajesh C, Teena R, Sunil K. Brucellosis: An update review of the disease. Indian J Anim Sci. 2013;83(1):3-16.

5. Shaalan MA, Memish ZA, Mahmoud SA, Alomari A, Khan MY, Almuneef M, et al. Brucellosis in children: clinical observations in 115 cases. Int J Infec Dis. 2002;6(3):182-6.

6. Roushan MRH, Amiri MJS, Laly A, Mostafazadeh A, Bijani A. Follow-up standard agglutination and 2-mercaptoethanol tests in 175 clinically cured cases of human brucellosis. Int $J$ Infect Dis. 2010;14(3):e250-3.

7. Rahamathulla MP. Seroprevalence of Human Brucellosis in Wadi Al Dawaser region of Saudi Arabia. Pak J Med Sci. 2018;35(1):129-35.

8. Prakash P, Bhansali S, Gupta E, Kothari D, Mathur A, Ambuwani S. Epidemiology of brucellosis in high risk group \& PUO patients of western-Rajasthan. Indian J Community Med. 2012;3(1):61-5.

9. Muloki HN, Erume J, Owiny DO, Kungu JM, Nakavuma J, Ogeng $\mathrm{D}$, et al. Prevalence and risk factors for brucellosis in prolonged fever patients in post-conflict Northern Uganda. Afr Health Sci. 2018;18(1):22-8

10. Metri BC, Mahesh CB, Jyothi P, Lava R, Basavarajappa, Hanumanthappa AR. Seroprevalence of Brucellosis In Davangere Karnataka. $J$ Clin Diag Res. 2011;5(1):41-4.

\section{Author biography}

\section{Pradeep Kumar Student}

Shweta R Sharma Assistant Professor

Umar Farooq Professor\& HOD

Sudhir Singh Associate Professor

Vasundhara Sharma Assistant Professor

Imran Ahamad Senior Resident

Shivendra Mohan Demonstrator

Cite this article: Kumar P, Sharma SR, Farooq U, Singh S, Sharma V, Ahamad I, Mohan S. Occurrence of brucellosis in patients of pyrexia of unknown origin. IP Int J Med Microbiol Trop Dis 2020;6(2):113-116. 